\title{
EEG MATURATIONAL CHANGES IN PARTIAL EPILEPSIES
}

Changes and migration of EEG foci with age in 208 patients with childhood partial epilepsy followed for more than 3 years are reported from the Department of Pediatrics, Toyama Medical and Pharmaceutical University, Toyama City, Japan. Frontal and central foci were frequent before school age and after adolescence. Temporal foci peaked around adolescence, and occipital foci between 3 to 7 years. Parietal foci were rare at all ages. Migration of EEG foci in 39\% of patients was frequent at early school age (anterior to posterior) and preadolescence (posterior to anterior). The migration of EEG foci was not accompanied by changes in seizure patterns or frequency. (Konishi T, Naganuma $\mathrm{Y}$, Hongou $\mathrm{K}$ et al. Changes in EEG foci with age in childhood partial epilepsies. Clin Electroencephalogr July 1994;25:104-109). (Reprints: Tohru Konishi MD, Dept of Pediatrics, Faculty of Medicine, Toyama Medical and Pharmaceutical University, 2630 Sugitani, Toyama City 930-01, Japan).

COMMENT. Migration of EEG foci in this study occurred with symptomatic partial epilepsies as well as idiopathic seizures. This phenomenon is not necessarily indicative of a benign epilepsy but reflects maturational changes in the brain, similar to those that characterize the changing patterns of seizures and EEG with infantile spasms and other epileptic syndromes. The authors suggest that this migration is an epiphenomenon on the scalp EEG since changes in clinical seizures were not observed.

\section{EEG DISCONTINUITY AND NEONATAL ACIDOSIS}

The effect of acidosis on cerebral function was evaluated by computerized online EEG monitoring in 14 ventilated preterm infants less than 32 weeks' gestation at the Department of Paediatrics and Neonatal Medicine, Royal Postgraduate Medical School, Hammersmith Hospital, London, UK. All episodes of acidosis were associated with periods of EEG discontinuity (attenuated activity between bursts of EEG activity). In more than half a pH $<7.20$ was recorded, but EEG changes were noted even with acidosis levels of $\mathrm{pH}$ 7.20-7.25. In 21 of 32 episodes, EEG activity returned to pre-acidosis levels after therapy for acidosis. Duration of EEG discontinuity was related to severity of acidosis. Recovery of EEG was quickest for pure-respiratory acidoses that required only simple ventilatory adjustments. (Murdoch Eaton DG, Dubowitz V et al. Reversible changes in cerebral activity associated with acidosis in preterm neonates. Acta Paediatr May 1994;83:486-92). (Respond: Dr D Murdoch Eaton, Academic Unit of Paediatrics, D Floor, Clarendon Wing, The General Infirmary at Leeds, Belmont Grove, Leeds LS2 9NS, UK).

COMMENT. Increases in the amount of discontinuity in the EEG of infants may reflect disturbances in neonatal cerebral function caused by acidosis, hypoxia, or low cerebral blood flow. These changes are reversible in contrast to EEG suppression associated with hemorrhage that is persistent. EEG discontinuity may be used as a sign of acute neonatal cerebral dysfunction requiring immediate therapeutic intervention. This apears to be the first report of EEG changes in the neonate associated with acidosis. 